\title{
Peculiarities of neutron scattering by order-disorder antiferroelectrics
}

\author{
S.Stamenković \\ The "Vinča" Institute of Nuclear Sciences, \\ Belgrade, Serbia, Yugoslavia
}

Received August 7, 2002, in final form August 25, 2002

\begin{abstract}
Using the dynamical proton (pseudospin)-heavy ion (lattice) model and the Green function formalism, the effective total differential cross sections for both coherent and incoherent neutron scattering are derived. The characteristic interference effects due to both tunnelling of light ions (protons) and mixed proton-heavy ion scattering processes are expressed by corresponding pseudospin and pseudospin-phonon form-factors. The scattering intensity pattern in antiferroelectric phase is accomplished by polar modulation effects and additional interference in the cross sections. The theoretical predictions are related to experimental studies of ADP, Rochelle salt and $\mathrm{NaNO}_{2}$. Their possible application to test the coexistence of (anti)ferroelectric and superconducting phases in high- $T_{\mathrm{c}}$ copper-oxide superconductors is briefly discussed too.
\end{abstract}

Key words: neutron scattering, antiferroelectric, pseudospin-phonon model

PACS: $61.12 . B t$

\section{Introduction}

Like in order-disorder ferroelectrics, a characteristic feature of the theoretical description of the dynamics of order-disorder antiferroelectrics, particularly of neutron scattering on such crystals, is the strong anharmonicity of the local one-particle potential as apparently present in them. In the most simple approach both systems are characterized by the collective motion of ions in a local potential with the two minima of equal or different depth and equilibrium positions $\mathbf{r}=\mu \mathbf{l}_{\mu}(\mu= \pm)$. Such a physical image is employed in describing various order-disorder ferroelectrics [for example, potassium dihydrogen phosphate, $\mathrm{KH}_{2} \mathrm{PO}_{4}(\mathrm{KDP})$, triglycine-sulphate acid (TGS), sodium nitrite $\left(\mathrm{NaNO}_{2}\right)$, etc.] and antiferroelctrics [such as ammonium dihydrogen phosphate, $\mathrm{NH}_{4} \mathrm{H}_{2} \mathrm{PO}_{4}$ (ADP), Rochelle salt, $\mathrm{NaKC}_{4} \mathrm{H}_{4} \mathrm{O}_{6} \cdot 4 \mathrm{H}_{2} \mathrm{O}$ (RS), trihydrogen selenite, $\mathrm{CsH}_{3}\left(\mathrm{SeO}_{3}\right)_{3}$ and others], together with compounds isomorphic and related to them $[1-5]$. In the more traditional approach the small distances $\mathbf{l}_{ \pm}$ 
may just represent stable configurations or equilibrium positions of ions, depending on how they change owing to statistical or quantum (tunnelling) jumps inside the one-particle potential. The definition of $\mathbf{l}_{ \pm}$will be more general, however, if it is related to equilibrium positions of all the ions in a primitive cell, in accordance with the notion of local normal coordinate $[1,5]$ (cf. $[6,7]$ ). Within the framework of such a generalized scheme the local normal coordinates practically coincide with the displacements of those ions that are active at phase transition.

It is well known that the disordering motion of active ("light") ions, or of all the ions in the primitive cell, is described by pseudospin variables as introduced by De Gennes and originally associated with the tunnelling of protons in their local doublewell potentials $[7,8]$, as probably the main characteristic of the hydrogen-bonded ferroelectrics. Shortly, the dynamical model of the pseudospin-phonon interaction (i.e. coupled proton-lattice model) was formulated $[9,10]$ and later on, being developed by many authors $[2,3,7,11-17]$ it has been widely applied in various order-disorder systems, predominantly in the studies of hydrogen-bonded ferroelectrics and antiferroelectrics (cf. [1-7] and references therein).

It is generally believed that order-disorder antiferroelectrics can be well represented by the physical situation as apparently encountered in corresponding hydrogen-bonded systems $[1,4,5]$. Such compounds are typified by ADP-crystal as entirely KDP-type antiferroelectric, and we shall deal with them predominantly throughout this paper. The treatment will be extended to another representative hydrogenbonded antiferroelectric, to Rochelle salt, as characterized by an asymmetric doublewell single-particle potential and rather resembling the features of a ferroelectric compound $[2,18]$.

The majority of theoretical examinations in the above mentioned representative antiferroelectrics are applicable to a number of other systems [like $\mathrm{NaH}_{3}\left(\mathrm{SeO}_{3}\right)_{2}$, $\mathrm{NH}_{4} \mathrm{HSO}_{4}$, etc.], including those in which the tunnelling of active atoms is small (as in deuterated compounds) or can be neglected (cf. [1-5]). In such cases we are left with the dynamic picture of a kinetic Ising type being replaced by a pure relaxational one. Note that in this context a very intriguing situation is present in $\mathrm{NaNO}_{2}$ : the ferroelectric phase is separated from the paraelectric one by a very narrow sinusoidal antiferroelectric phase (cf. [1,4]). In the context of the neutron dynamical neutronography, among many unexplained physical characteristics of $\mathrm{NaNO}_{2}$ as a typical non-hydrogenous order-disorder ferroelectric, the appearance of a sinusoidal antiferroelectric phase particularly deserves a more complete theoretical as well as a non-conventional and more precise experimental research.

Based on the relatively recent experimental (by neutron scattering and other techniques) and theoretical analysis [19-21] (and references therein) on possible lattice instability associated with a split position of the "apical" oxygen atoms (denoted as $\mathrm{O} 4$ ) within their double-well local potentials in $\mathrm{YBa}_{2} \mathrm{Cu}_{3} \mathrm{O}_{7}$ superconductor, for such and related compounds one can perform the analogous derivation of neutron scattering functions within the framework of a generalized pseudospin-phonon model of the Rochelle salt type. The possible application of such adjusted expressions for testing the coexistence of superconducting and (anti)ferroelectric phases in high- $T_{\mathrm{c}}$ 
copper-oxide superconductors is proposed and briefly commented in the end of the paper.

From a formal point of view derivation of the differential neutron scattering cross sections for order-disorder antiferroelectrics is connected with peculiarities of computing the corresponding particle-density correlation functions for discrete (pseudospin) and harmonic-like (phonon) variables taking into account the coupling between the pseudospin and phonon modes in the system (cf. [5] and references therein). One must also bear in mind the specific features of the symmetry aspects of the analysis of scattering cross sections in connection with the physical meaning of the pseudospin operator and its being different from the real spin with respect to the time reversal [22].

To clearly present the derivation of expressions for the scattering cross sections (for coherent, incoherent, elastic and inelastic scattering) of neutrons on antiferroelectrics of the order-disorder type and to better comprehend the features peculiar to such scattering, we shall first outline the dynamics of antiferroelectrics with hydrogen bonds by making use of the special case of ADP and RS crystals.

\section{Basic dynamics in the framework of pseudospin-phonon model}

Antiferroelectrics of ADP-type and antiferroelectrics of the compounds isomorphic to them are of great interest due to the relative simplicity of their structure (although there are enough atoms per unit cell) and their availability, which permits to investigate the general properties of such hydrogen-bonded crystals. From a practical standpoint they can be grown easily and being in general of high quality they yield various technological applications [1]. Besides, such crystals exhibit a number of features relevant to the theory of phase transitions, particularly being of interest in the case of order-disorder systems with an antiparallel arrangement of electric dipoles.

The phase transition in ADP has been known for almost 65 years, although for a long time it was thought to be basically different from the KDP-type ferroelectrics. The ADP structure was established by X-rays and neutron diffraction both in paraelectric (tetragonal) and antiferroelectric (orthorhombic) phases (cf. $[1,2]$ ). The survey of structural, symmetry and physical properties in general, e.g. the corresponding data for ADP and all KDP-type ferroelectrics, can be found in special books, reviews or articles, for example in monographs and papers cited herein $[1-5,22]$ (and references therein). Therefore, only the main physical characteristics of ADP are quoted hereto as mostly important for the following. Thus, the primitive cell in the high temperature phase $\left(T>T_{\mathrm{N}} \simeq 150 \mathrm{~K}\right)$ contains two formula units, the unit cell (with four formula units) in turn being doubled below the phase transition. In the antiferroelectric (antipolar) phase, unlike KDP, one "upper" and one "lower" proton sites are filled in off-center positions nearer to an arbitrary $\mathrm{PO}_{4}$ group. While the hydrogen bonds are always nearly perpendicular to the c-axis, the displacements of ammonium and phosphate groups below the critical temperature are in the $a-b$ 
planes resulting in an antipolar ordering (in the $c$-direction) of these neighboring groups. In turn, either the $a$ - or $b$-directions can become antiferroelectric axes below $T_{\mathrm{N}}$. The principle role of protons is manifested in the large isotope effect (for instance, in sharp changes of the transition temperature and the tunnelling energy when hydrogen is substituted by deuterium: $\left.T_{\mathrm{N}}^{\mathrm{D}} \simeq 242 \mathrm{~K}, 2 \Omega_{\mathrm{D}} \simeq(0.60 \div 0.70) 2 \Omega_{\mathrm{H}}\right)$ $[1-3]$.

Rather more than in case of the hydrogen-bonded ferroelectrics, the description and experimental investigations of the complete dynamics of isomorphic antiferroelectric compounds represents an extremely difficult task [1,4]. For instance, a determination of the actual atomic movements in the antiferroelectric mode would require a fit of 20 amplitude parameters $[1,5]$. In this respect, there are not too many papers in the field of research concerned. Although being not too much noticeable in the current literature, extension of the pseudospin [23,24] and dynamical (coupling) tunnelling proton-lattice [25] models in the antiferroelectric context had been probably developed long time ago. Later on these models were applied in the corresponding theory of neutron scattering, by ferroelectrics and antiferroelectrics (mainly by ADP) [26,27] (cf. [5]).

As it was mentioned in the previous section, from the model point of view and its efficiency as well, i.e. to overcome the standard difficulties in solving the eigenvalue problem whereby applying the symmetry analysis (cf. [5]), it is convenient to utilize the representation of local normal coordinates: the first one is associated with the tunnelling motion of all ions (mainly of protons) in each unit cell $(n=1, \ldots, N)$, being further related to the pseudospin variables $S_{n}^{\alpha}(\alpha=x, y, z)$ and the corresponding mode of energy $\varepsilon_{q}=\hbar \omega_{q}$; the other two $(j=1,2)$ describe phonon vibrations (in the $a-b$ planes), i.e. the modes of the same frequencies $\left(\omega_{q 1}=\omega_{q 2}=\lambda_{q}\right)$ but of different polarizations $\left(\mathbf{e}_{q 1} \neq \mathbf{e}_{q 2}\right)$, both perpendicular to the c-axis. Thus, taking advantage of local normal coordinate and pseudospin representations the Hamiltonian of the system can be written in the convenient Fourier-transformed form [9-11] as specified for three interacting modes [25],

$$
\mathrm{H}=-\sqrt{N}\left(2 \Omega S_{0}^{x}+\Delta S_{0}^{z}\right)-\sum_{q} J_{q} S_{q}^{z} S_{-q}^{z}-\sum_{q j}\left[\Phi_{q j}\left(b_{q j}^{+}+b_{-q j}\right) S_{-q}^{z}+\hbar \lambda_{q j} b_{q j}^{+} b_{q j}\right] .
$$

Here, the transverse field $2 \Omega=\langle R n|V(n)| n L\rangle$ describes tunnelling of the $n$-th primitive cell from a state of the "left-hand", $|n L\rangle$, to a state of the "right hand" configuration, $|n R\rangle$, and $\Delta$ is the parallel field as a measure of the asymmetry of a local double-well potential; $J_{q}$ is the Fourier transform of the effective exchange interaction between the cells $n$ and $n^{\prime}, \Phi_{q j}$ is the Fourier transform of the proton (pseudospin) - heavy ion interaction (for phonon branches $j=1,2$ ). The Coulomb nature of the interaction $\Phi_{q j}$ was dealt with in $[10,11]$. Particularly, it was represented as the difference of two terms $T_{q j}( \pm)$ corresponding, respectively, to the left $(-)$ and right $(+)$ minima of a hydrogen bond [11],

$$
\Phi_{q j}^{r f}=\left[T_{q j}^{r f}(+)-T_{q j}^{r f}(-)\right]=\Phi_{-q j}^{r f *},
$$

where $T_{q j}^{r f}( \pm)$ are given by almost exact expressions in the convenient lattice-ion-site summation form. In the continuous approximation $\left(\mathbf{R}_{n r} \rightarrow \mathbf{R}\right), \Phi_{q j}^{r f}$ (indexes $r$ and 
$f$ labelling the light and heavy ions, respectively, in a primitive cell) can be cast in the form which is more suitable for particular evaluations [28],

$$
\Phi_{q j}^{r f}=\mathrm{i} \frac{8 \pi e^{* 2}}{V_{0}|\mathbf{q}|^{2}} \sqrt{\frac{\hbar}{2 m_{f} \omega_{q j}}}\left(\mathbf{q} \cdot \mathbf{e}_{\mathrm{qj}}^{\mathrm{f}}\right)\left(\mathrm{e}^{\mathrm{iq} \mathbf{l}_{r-}}-\mathrm{e}^{-\mathrm{i} \mathbf{q} \mathbf{l}_{r+}}\right) \mathrm{e}^{\mathrm{i} \mathbf{q}\left(\mathbf{R}_{\mathrm{o} r}-\mathbf{R}_{\mathrm{o} f}\right)} .
$$

Here $e^{*}$ is the effective charge measuring the Coulomb interaction between hydrogens and heavy ions, and $V_{0}$ is the volume of the unit cell, while $\mathbf{R}_{o r}$ and $\mathbf{R}_{\text {of }}$ denote the positions of light and heavy ions in it, respectively.

In the local normal coordinate representation the above quantity (3) acquires a simpler form,

$$
\Phi_{q j}=\mathrm{i} \frac{8 \pi e^{* 2}}{V_{0}|\mathbf{q}|^{2}} \sqrt{\frac{\hbar}{2 m \omega_{q j}}}\left(\mathbf{q} \cdot \mathbf{e}_{\mathrm{qj}}\right)\left(\mathrm{e}^{\mathrm{i} \mathbf{q} \mathbf{l}_{-}}-\mathrm{e}^{-\mathrm{i} \mathbf{q} \mathbf{l}_{+}}\right) \mathrm{G}^{*}(\mathbf{q}),
$$

where $G(\mathbf{q})=\sum_{\mathrm{f}} \exp \left(\mathbf{i q} \mathbf{R}_{\mathrm{o} f}\right)$ is the structure factor of heavy ions in the primitive cell and $m$ is the reduced mass of heavy-ion complex $\left[\mathrm{NH}_{4}-\mathrm{PO}_{4}\right]$.

The dispersion law for two mixed modes in the model (1) was obtained by several equivalent methods. Firstly, the Mori method of moments was used [9], then the method of equations of motion [10] and, eventually, the method of Green functions was applied $[11,29]$, of which we shall further take advantage.

In the pseudospin space it is convenient to pass from the $x y z$-coordinate system to the coordinates $\xi y \zeta$, such that the average value $\langle\mathbf{S}\rangle$ is directed along the $O \zeta$-axis so that values $\left\langle S^{\zeta}\right\rangle=\left\langle S^{y}\right\rangle=0$. The transformation has the form [25]

$$
S_{q}^{x}=S_{q}^{\zeta} \sin \varphi+S_{\left(q-q_{\mathrm{B}}\right)}^{\xi} \cos \varphi ; \quad S_{q}^{z}=S_{\left(q-q_{\mathrm{B}}\right)}^{\zeta} \cos \varphi+S_{q}^{\xi} \sin \varphi ;
$$

the critical wave vector $\mathbf{q}_{\mathrm{B}}$ is the wave vector on the boundary of the Brillouin zone (BZ) and $\varphi$ is the angle between the $O z$ - and $O \zeta$-axes,

$$
\varphi=\arctan \left(H_{x} / H_{z}\right)
$$

The transformation (5) means that in one sublattice the rotation angle is $\varphi$ while in the other one is $(\pi-\varphi)$. Note that $S_{q+q_{\mathrm{B}}}^{\alpha}=S_{q-q_{\mathrm{B}}}^{\alpha}$ since $2 \mathbf{q}_{\mathrm{B}}$ is the reciprocal lattice vector.

The molecular field components in equation (6) are:

$$
\begin{aligned}
& H_{x}=2 \Omega ; \quad H_{z}=2 \bar{J}_{q_{\mathrm{B}}}\left\langle S^{z}\right\rangle+\Delta ; \\
& \bar{J}_{q}=J_{q}+\sum_{j}\left|\Phi_{q j}\right|^{2} / \lambda_{q j} ; \\
& \left\langle S^{\alpha}\right\rangle=\left(H_{\alpha} / 2 H\right) \tanh (H / 2 T), \quad(\alpha=x, z) ; \quad H=\left[H_{x}^{2}+H_{z}^{2}\right]^{1 / 2} ;
\end{aligned}
$$

$\bar{J}_{q}$ is the Fourier transform of the effective pseudospin interaction; $\left\langle S^{x}\right\rangle$ and $\left\langle S^{z}\right\rangle$ are related as $\left\langle S^{x}\right\rangle H_{z}=\left\langle S^{z}\right\rangle H_{x}$. For the further computation of the neutron scattering 
cross sections the necessary Green functions in the random-phase approximation [11,25,29] (cf. [5]) are stated as follows:

$$
\begin{aligned}
& G^{\xi \xi}(\mathbf{q}, \omega)=-\left\langle\left\langle S_{q}^{\xi} \mid S_{-q}^{\xi}\right\rangle\right\rangle_{\omega}=\frac{-H\left\langle S^{\zeta}\right\rangle\left(\omega^{2}-\lambda_{q}^{2}\right)}{\left(\omega^{2}-E_{q j+}^{2}\right)\left(\omega^{2}-E_{q j-}^{2}\right)}, \\
& G^{j \xi}(\mathbf{q}, \omega)=-\left\langle\left\langle A_{q j} \mid S_{-q}^{\xi}\right\rangle\right\rangle_{\omega}=\frac{-2 \Phi_{q j}^{*} H \lambda_{q}\left\langle S^{\zeta}\right\rangle \sin \varphi}{\left(\omega^{2}-E_{q j+}^{2}\right)\left(\omega^{2}-E_{q j-}^{2}\right)}, \\
& G^{j j}(\mathbf{q}, \omega)=-\left\langle\left\langle A_{q j} \mid A_{-q j}\right\rangle\right\rangle_{\omega}=\frac{-2 \lambda_{q}\left(\omega^{2}-\omega_{q}^{2}\right)}{\left(\omega^{2}-E_{q j+}^{2}\right)\left(\omega^{2}-E_{q j-}^{2}\right)},
\end{aligned}
$$

where $A_{q j}=b_{q j}+b_{-q j}^{+}$and the frequency of the pseudospin mode is given by

$$
\omega_{q}^{2}=H^{2}-2 H\left\langle S^{\zeta}\right\rangle J_{q} \sin ^{2} \varphi
$$

The frequencies of coupled pseudospin-phonon modes are determined by the poles of any one of the Green functions (10)-(12):

$$
E_{q j \pm}^{2}=\frac{\lambda_{q}^{2}+\omega_{q}^{2}}{2} \pm\left[\left(\frac{\lambda_{q}^{2}-\omega_{q}^{2}}{2}\right)^{2}+2 H \lambda_{q}\left\langle S^{\zeta}\right\rangle \sum_{j^{\prime}}\left|\Phi_{q j^{\prime}}\right|^{2} \sin ^{2} \varphi\right]^{1 / 2}
$$

Here the parameter $\left\langle S^{\zeta}\right\rangle$ is calculated from the mean field equations (7)-(9), or, to be more precise, $\left\langle S^{\zeta}\right\rangle=1 / 2-N^{-1} \sum_{q}\left\langle S_{-q}^{-} S_{q}^{+}\right\rangle$, where the correlator is computed with the aid of the Green function $\left\langle\left\langle S_{-q}^{-} \mid S_{q}^{+}\right\rangle\right\rangle[11]$.

As one can see from equation (14), with account of equations (6)-(9) and equation (13), the pseudospin-like mode $\left(E_{q-} \equiv E_{q j-}\right)$, often named "antiferroelectric", condenses at the edge of BZ, while the frequencies of the phonon-like ones $\left(E_{q j+} ; E_{q 1+}=E_{q 2+}\right)$ remain finite attaining their minimum when $T \rightarrow T_{\mathrm{N}}$ from above (cf. [25]). This indicates that at $T=T_{\mathrm{N}}$ and for $\mathbf{q}=\mathbf{q}_{\mathrm{B}}$ the instability of the paraelectric phase occurs; the antiferroelectric phase begins to develop and the unit cell is doubled (BZ becomes twice smaller). Therefore, all functions of quasimomentum should be referred to the new BZ. Caused by such a reduction, all modes become doubled $\left(E_{\left(q+q_{\mathrm{B}}\right) j \pm}\right.$ appear in common to $\left.E_{q j \pm}\right)$ and all summations over $q$, $\sum_{q} f(q)$, have to be substituted by $\left[\sum_{q} f(q)+f\left(q+q_{\mathrm{B}}\right)\right][25]$. The antiferroelectric (antipolar) phase is represented by the pseudospin (polarization) modulation $[2,5]$,

$$
\left\langle S_{n}^{z}\right\rangle=\exp \left(\mathbf{i q}_{\mathrm{B}} \cdot \mathbf{n}\right)\left\langle\mathrm{S}^{\mathrm{z}}\right\rangle,
$$

meaning that a sinusoidal structure below $T_{\mathrm{N}}$ is stable rather than the homogeneous one $\left(\left\langle S_{i}^{z}\right\rangle=\left\langle S^{z}\right\rangle\right)$. In turn all three (i.e. two) modes $E_{q j \pm}(14)$ depend on the sublattice mean values (labelled by index $r=1,2),\left\langle S_{n r}^{z}\right\rangle=\left\langle S_{r}^{z}\right\rangle=\left\langle S_{1,2}^{z}\right\rangle= \pm\left\langle S^{z}\right\rangle$; the corresponding $z$-components of the fields $H_{r}$ are given by:

$$
\begin{aligned}
& H_{1,2}^{z}=2 \bar{J}_{11}\left\langle S_{1,2}^{z}\right\rangle+\bar{J}_{12}\left\langle S_{2,1}^{z}\right\rangle \pm \Delta ; \\
& \bar{J}_{r r^{\prime}}=\sum_{n r^{\prime}} \bar{J}_{i n}^{r r^{\prime}}, \quad \bar{J}_{11}=\bar{J}_{22}, \quad \bar{J}_{12}=\bar{J}_{21} .
\end{aligned}
$$


In the absence of an external field $\left(\bar{E}_{0}\right)$, for all temperatures there holds $\left\langle S_{1}^{z}\right\rangle=$ $-\left\langle S_{2}^{z}\right\rangle$. It should be emphasized that in the case of Rochelle salt $\left\langle S_{1}^{z}\right\rangle \neq-\left\langle S_{2}^{z}\right\rangle$ resulting in a net polarization in the ferroelectric-like (polarized) phase $T_{\mathrm{c} 1}<T<$ $T_{\mathrm{c} 2}$. The relevant numerical calculations of mean values $\left\langle S_{1}^{z}\right\rangle$ and $\left\langle S_{2}^{z}\right\rangle$ including two relaxation times, $\tau_{q \pm}$, corresponding to two (degenerated) modes $\omega_{q \pm}=\omega_{q}, \omega_{q+q_{\mathrm{B}}}$ (equation (13)) for deutered RS have been done whereby a good agreement with experimental data has been obtained [18] (cf. [2]).

A more pronounced dynamical theory of antiferroelectric phase transitions in crystals with asymmetric hydrogen bonds (or other single-particle potentials of a double-well type) and several proton sublattices as well, has been developed and applied to Rochelle salt-type compounds [15]. It should be stressed that apart from a series of original derivations, numerical estimations, discussions and auxiliary details in widely conceived content of qualitative and quantitative predictions, in the above mentioned paper [15] the frequencies of the mixed "soft phonon-proton" and "pseudo-soft proton-phonon" modes in RS are calculated within the suitably generalized pseudospin-phonon model. Without going too much into details, several crucial points should be outlined. First, the ferroelectric dipole moments in the system are associated with the ordering of protons on hydrogen bonds $\mathrm{O}_{(1)}-\left(\mathrm{H}_{2} \mathrm{O}\right)_{(10)}$ or with hydroxyl groups $(\mathrm{OH})_{(5)}$ within their local potentials (the ordering elements of another more complex nature is possible). Since an isomorphous substitution (in a very small extent) of $\mathrm{Rb}, \mathrm{Tl}$ and $\mathrm{NH}_{4}$ for $K$ eliminates the net-polarized phase, it seems that $K$-atoms (alone or with their surroundings) play an active role in the phonon subsystem and in the proton (pseudospin)-heavy ion (phonon) interaction as the ones responsible for the phase transitions (at $T_{\mathrm{c} 1}$ and $T_{\mathrm{c} 2}$ ). It is interesting that in comparison with ADP, the antiferroelectric-like mode in RS is of the phonon-protonlike (displacive) type, while the proton-phonon-like (pseudo-soft) one remains stable by approaching both the critical temperature and the critical wave vector. Moreover, the temperature behavior of the soft mode is essentially determined by the competition of two "fields": the transverse field of tunnelling and the parallel field of asymmetry, since both of them present the temperature dependent characteristics of the local particle (site) potential. Besides, as usual, at temperatures close to $T_{\mathrm{c}}$, the damping of the soft mode may appear to be large since the dispersion of the dielectric permeability $\varepsilon(\omega)$ is rather of a relaxation type. It is quite intriguing that the parameters of the phonon subsystem also change with deuteration. Therefore, the experimental study of all these predictions in general and particularly using neutron scattering, would be of great interest.

Qualitative and quantitative estimations of the damping factors, $\Gamma_{q \pm}$, of the respective mixed modes (14) as functions of temperature and of the wave vector represent one of the most important problems in the dynamics of ferroelectrics and antiferroelectrics with hydrogen bonds [5]. Of utmost interest is the study of critical damping which has not been explained completely so far. Numerous experiments utilizing various methods of measurements show that, as the critical point of the phase transition $T_{\mathrm{c}}\left(T_{\mathrm{N}}\right)$ is approached, the damping factor $\Gamma_{q-}$ increases significantly, and thus the soft mode becomes relaxational. Theoretical analysis of such a large damp- 
ing isn't easy due to the strong interaction between elementary excitations and large fluctuations of the order parameter in the system near $T_{\mathrm{c}}$.

The simplest way is to take the damping into account phenomenologically by substituting the time dependent phase factor $\exp \left( \pm \mathrm{i} E_{q \pm} t-\Gamma_{q \pm}|t|\right)$ for $\exp \left( \pm \mathrm{i} E_{q \pm} t\right)$ in the corresponding pseudospin correlators. Then the $\delta$-function will be replaced by the Lorentz curve, so that information can be derived from the neutron energy distribution concerning damping factors $\Gamma_{q \pm}$ of the respective modes. Within the framework of the pseudospin-phonon model, the damping was included in $[27,30]$. Then, it can be chosen to be such that the behavior of the modes is either weakly damping or relaxational. Thus, for example, when the damping is independent of the frequency, the soft mode is assumed to be of a relaxational nature in the close vicinity of $T_{\mathrm{c}}$. More sophisticated approaches to the pseudospin-phonon dynamics utilizing the method of Green functions and of relaxational functions lead to a more complicated behavior of the modes (of their frequencies and widths). In this approach, under certain conditions (definite values of the model parameters) in the region $T \geqslant T_{\mathrm{c}}$, the central peak is also reproduced with the aid of approximations being beyond the framework of random phase approximation and also by utilizing numerical calculations [7,31] (cf. [5] and references therein).

\section{Differential cross sections}

Most theoretical works on neutron scattering in KDP hydrogen-bonded ferroelectrics were performed by applying the Blinc - de Gennes model in which the effect of heavy ion vibrations on the proton subsystem is taken into account only by renormalization (due to pseudospin-phonon interaction) (cf. [5]). Within the framework of such an approach inelastic incoherent scattering has been considered in $[8,30]$, while inelastic coherent scattering in pseudospin-phonon model was proposed in ref [29] and subsequently it was studied in greater detail in ref [27]. Such a theory of the elastic and one-quantum inelastic neutron scattering was formulated in order to take into account the real structure of hydrogen-bonded ferro- and antiferroelectrics. In this fashion, the interference scattering term due to the light ion (proton)-heavy ion interaction has been taken into account as well. Moreover, the account was taken of the adiabatic change of positions of the minima of the double well, considering them as the ones "attached" to the nearest (i.e. the nearest oxygen) ions [27]. However, in the local-pseudospin-coordinate representation, from a formal mathematical point of view, the scattering functions are greatly reduced since the pseudospin operator acquires the physical meaning of a cell variable. The respective pseudospin-form factors are refined correspondingly, while the contribution of critical (pseudospin-like antiferroelectric hereto) mode (indicated by the index $c$ ) will be excluded from the scattering function for the subsystem of heavy ions [5,7]. By ignoring the usually small asymmetry of the one-cell potential, the scattering functions are significantly simplified in the approximation of interaction of the critical mode with two optical phonon modes [as proposed by Stasyuk and Levitskii [25], equation (1)] (cf. [5]). 
By using the standard notation and slightly modified Green functions as given by equations (10)-(12), the total differential cross section for coherent (ch) and incoherent (ic) scattering of neutrons by order-disorder antiferroelectrics is cast in the form [33] (cf. [5,27])

$$
\frac{1}{N}\left(\frac{\mathrm{d}^{2} \sigma}{\mathrm{d} \Omega \mathrm{d} E}\right)=S_{\mathrm{el}}(\kappa) \delta(\omega)+\frac{p}{p_{o}}\left[S^{-}(\kappa, \omega)+S^{+}(\kappa, \omega)\right],
$$

where $\hbar \kappa=\mathbf{p}_{o}-\mathbf{p}, \hbar \omega=E_{o}-E$ are the neutron transferred momentum and energy, $\mathbf{p}_{\mathbf{o}}, E_{o}$, and $\mathbf{p}, E$ being initial and final momenta and energies, respectively. The first term in equation (18) is referred to the elastic differential cross section, while the scattering functions $S^{\nu}(\kappa, \omega)(\nu=-,+)$ describing the one-quantum inelastic scattering correspond to $E_{q \nu}$ modes $\left(E_{q-}, E_{q+}=E_{q j+}\right)$. Upon integrating over the energies of the scattered neutrons the following expressions for the angular distribution of the elastic coherent and incoherent scattering are obtained:

$$
\begin{aligned}
\frac{1}{N}\left(\frac{\mathrm{d} \sigma_{\mathrm{ch}}}{\mathrm{d} \Omega}\right)= & N \Delta(\kappa)\left[\left(b_{\mathrm{ch}}^{\mathrm{c}}\right)^{2}\left|\mathrm{e}^{-W_{\kappa}^{\mathrm{c}}} F_{\mathrm{c}}(\kappa)\right|^{2}+\left|\sum_{j} \mathrm{e}^{-W_{\kappa}^{j}} b_{\mathrm{ch}}^{j} G(\kappa)\right|^{2}\right. \\
& +\sum_{j} b_{\mathrm{ch}}^{\mathrm{c}} b_{\mathrm{ch}}^{j} \mathrm{e}^{-W_{\kappa}^{\mathrm{c}}} \mathrm{e}^{-W_{\kappa}^{j}} \operatorname{Re}\left[F_{\mathrm{c}}^{*}(\kappa) G(\kappa)\right] \\
\frac{1}{N}\left(\frac{\mathrm{d} \sigma_{\mathrm{ic}}}{\mathrm{d} \Omega}\right)= & \left(b_{\mathrm{ic}}^{\mathrm{c}}\right)^{2}\left|F_{\mathrm{c}}(\kappa) \mathrm{e}^{-W_{\kappa}^{\mathrm{c}}}\right|^{2}+\sum_{j}\left(b_{\mathrm{ic}}^{j}\right)^{2} \mathrm{e}^{-2 W_{\kappa}^{j}}
\end{aligned}
$$

Here the variation of the neutron wave vector due to scattering satisfies the following conservation law:

$$
\Delta(\kappa)=\frac{1}{N} \sum_{n} \mathrm{e}^{\mathrm{i} \kappa \cdot \mathbf{n}}=\sum_{\mathbf{b}} \delta_{\kappa, \mathbf{b}},
$$

where the summation runs over the reciprocal lattice vectors b. Note once more that index $c$ (actually identical with the index $\nu \equiv-$ ) points to the effective oneparticle potential, while one must make the index $j$ correspond to the index $f$ (both, $c$ and $j$ are associated with the pseudospin-like and local normal coordinate as previously explained). The scattering amplitudes (i.e. the lengths) $b_{\mathrm{ch}}^{c, j}$ and $b_{\mathrm{ic}}^{c, j}$ herein and henceforth have to be referred to the amplitudes obtained as a result of averaging over the ions participating in the given mode vibrations $\left(E_{q-}\right.$ or $\left.E_{q j+}\right)$. The Debye-Waller factors, $\exp \left(-W_{\kappa}^{j}\right)$, are the usual phonon ones, while the factor $\exp \left(-W_{\kappa}^{\mathrm{c}}\right) \equiv \exp \left(-W_{\kappa}^{-}\right)$is more specific and corresponds to the pseudospin-like $\operatorname{mode}\left(E_{q c} \equiv E_{q-} \equiv E_{q j-}\right)[5,28]$ :

$$
\begin{aligned}
& W_{\kappa}^{\mathrm{c}}=\frac{1}{2} \sum_{\mu} W_{\kappa}^{\mathrm{c}}(\mu)=\frac{1}{2} \sum_{j, \mu} \sum_{q}\left|\Lambda_{q}^{c j}(\mu)\right|^{2} W_{\kappa}^{j}(q), \\
& W_{\kappa}^{j}(q)=\frac{1}{N} \frac{\left(\kappa \cdot \mathbf{e}_{\mathbf{q j}}\right)^{2}}{2 \pi m \omega_{j}(q)} \int_{0}^{\infty} \operatorname{coth}\left(\frac{\omega}{2 k T}\right) K_{j j}^{\prime \prime}(q, \omega) \mathrm{d} \omega .
\end{aligned}
$$


Here the coefficients $\Lambda_{q}^{c j}(\mu)$ come from an expansion of the momentary positions of double-well minima in power of heavy ion displacements (introducing boson operators $b_{q j}^{ \pm}$for phonons) [30]. As pointed out in [5,30], they can be expressed through the Fourier components of the pseudospin-phonon interaction $\Phi_{q j}(\mu)$, i.e. the terms $T_{q j}(\mu)$ in equation $(2)$ :

$$
\Lambda_{q}^{c j}(\mu) \equiv \Lambda_{q j}(\mu)=\frac{T_{q j}(\mu)}{2 k_{\mathrm{c}}}, \quad\left|\Lambda_{q}^{c j}(\mu)\right| \leqslant 1
$$

$k_{\mathrm{c}}=m_{\mathrm{c}} \omega_{\mathrm{c}}^{2}$ is the force constant of one of the two interacting harmonic oscillators at the $n$-th site $\left(m_{\mathrm{c}}\right.$ is the effective mass of all ions in the cell participating in the pseudospin mode, and $\omega_{\mathrm{c}}$ is the corresponding classical frequency of the oscillator). Otherwise in the adiabatic model adopted in [27] a strong correlation between the motion of light and heavy ions is assumed, i.e. $\left|\Lambda_{q j}(+)\right|=\left|\Lambda_{q j}(-)\right|=1$. However, this approximation is justified with difficulty in he case of long-wave phonons $[4,5]$. We note that in the Debye-Waller factor (22) which effectively takes account of the effect of vibrations of heavy ions on light particles, averaging is performed over $\mu=(+,-)$. In a more rigorous approach, factors such as $\exp \left(-W_{\kappa}^{c \mu}\right)$ should be retained under the sum sign over $\mu$ in the form factor (25).

The elastic pseudospin form factor $F_{\mathrm{c}}(\kappa)$ in equations (19) and (20) is given by $[27,30]$ (cf. [5])

$$
F_{c}(\kappa)=\frac{1}{2} \sum_{\mu}\left[\mathrm{e}^{\mathrm{i} \mu \kappa \cdot \mathbf{l}_{\mu}} \alpha_{\kappa \mu}+2\left\langle S^{x}\right\rangle \beta_{\kappa \mu}+2 \mu\left\langle S^{z}\right\rangle \alpha_{\kappa \mu} \mathrm{e}^{\mathrm{i} \mu \kappa \cdot \mathbf{l}_{\mu}}\right] \sum_{r} \mathrm{e}^{\mathrm{i} \kappa \cdot \mathbf{R}_{\mathrm{or}}},
$$

in which the asymmetry of double-well potential is taken into account in terms of $\mathbf{l}_{\mu}$. One of the distances $\left|\mathbf{l}_{\mu}\right|=l_{\mu}$ (say right, $\mu=+$ ) could be dependent on the asymmetry parameter, $B=\Delta+H_{z}$, simply as $l_{+}=\sqrt{l^{2}-\left(2 B / k_{\mathrm{c}}\right)}$ or on the parameter $H_{z}$ only, depending on the model chosen [33].

The scattering functions in equation (18)

$$
S^{\nu}(\kappa, \omega)=[1+n(\omega)] \sum_{q}\left[N \Delta(\kappa-\mathbf{q}) A_{\mathrm{ch}, q}^{\nu}(\kappa, \omega)+A_{\mathrm{in}, q}^{\nu}(\kappa, \omega)\right],
$$

are expressed through the pseudospin-like $\left(P_{q}^{\nu}(\kappa, \omega)\right)$, the phonon-like $\left(F_{q}^{\nu}(\kappa, \omega)\right)$ and the mixed-interacting $\left(I_{q}^{\nu}(\kappa, \omega)\right)$ terms:

$$
\begin{aligned}
& A_{\mathrm{ch}, q}^{\nu}(\kappa, \omega)=\left(a_{\mathrm{ch}}^{P}\right)^{2} P_{q}^{\nu}(\kappa, \omega)+\left(a_{\mathrm{ch}}^{F}\right)^{2} F_{q}^{\nu}(\kappa, \omega)+a_{\mathrm{ch}}^{P} a_{\mathrm{ch}}^{F} I_{q}^{\nu}(\kappa, \omega), \\
& A_{\mathrm{in}, q}^{\nu}(\kappa, \omega)=\left(a_{\mathrm{ic}}^{P}\right)^{2} P_{q}^{\nu}(\kappa, \omega)+\left(a_{\mathrm{ic}}^{F}\right)^{2} F_{q}^{\nu}(\kappa, \omega) ;
\end{aligned}
$$

here the squares of the scattering amplitudes contain the corresponding structure factor of the primitive cell:

$$
\begin{aligned}
& \left(a_{\mathrm{ch}}^{G}\right)^{2}=\left(b_{\mathrm{ch}}^{g}\right)^{2}\left|\sum_{d} \mathrm{e}^{\mathrm{i} \kappa \cdot \mathbf{R}_{\mathbf{d}}}\right|^{2}, \quad\left(a_{\mathrm{ch}}^{I}\right)^{2}=b_{\mathrm{ch}}^{g} b_{\mathrm{ch}}^{j} \sum_{r, f} \mathrm{e}^{\mathrm{i} \kappa \cdot\left(\mathbf{R}_{\mathbf{r}}-\mathbf{R}_{\mathbf{f}}\right)}, \\
& G=P, F ; \quad g=c, j ; \quad d=r, f
\end{aligned}
$$


and similarly for $\left(a_{\mathrm{ic}}^{G}\right)^{2}$;

$$
\begin{aligned}
P_{q}^{\nu}(\kappa, \omega)= & 4\left\{|F(\kappa)|^{2} \mathrm{e}^{-2 W_{\kappa}^{-}} K_{\nu}^{\xi \xi^{\prime \prime}}(\mathbf{q}, \omega)+\sum_{j}\left\{2 \operatorname{Re}\left[L_{j}^{*}(\kappa, \mathbf{q}) F(\kappa)\right] \mathrm{e}^{-2 W_{\kappa}^{-}} K_{\nu}^{j \xi^{\prime \prime}}(\mathbf{q}, \omega)\right.\right. \\
& \left.\left.+\frac{1}{4}\left|\tilde{F}_{j}(\kappa, \mathbf{q})\right|^{2} \mathrm{e}^{-2 W_{\kappa}^{-}} K_{\nu}^{j j^{\prime \prime}}(\mathbf{q}, \omega)\right\}\right\}, \\
F_{q}^{\nu}(\kappa, \omega)= & \sum_{j}\left|F_{o j}(\kappa, \mathbf{q})\right|^{2} \mathrm{e}^{-2 W_{\kappa j}} K_{\nu}^{j j^{\prime \prime}}(\mathbf{q}, \omega), \\
I_{q}^{\nu}(\kappa, \omega)= & \sum_{j} 2\left\{4 \operatorname{Re}\left[F^{*}(\kappa) F_{o j}(\kappa, \mathbf{q})\right] \mathrm{e}^{-W_{\kappa}^{-}} \mathrm{e}^{-W_{\kappa}^{j}} K_{\nu}^{j \xi^{\prime \prime}}(\mathbf{q}, \omega)\right. \\
& \left.+\operatorname{Re}\left[\widetilde{F}_{j}^{*}(\kappa, \mathbf{q}) F_{o j}(\kappa, \mathbf{q})\right] \mathrm{e}^{W_{\kappa}^{-}} \mathrm{e}^{-W_{\kappa}^{j}} K_{\nu}^{j j^{\prime \prime}}(\mathbf{q}, \omega)\right\} .
\end{aligned}
$$

In the above expressions (28), the imaginary parts of the Green functions $K_{\nu}^{\xi \xi^{\prime \prime}}, K_{\nu}^{j \xi^{\prime \prime}}$ and $K_{\nu}^{j j^{\prime \prime}}$ are determined by the following expressions:

$$
\begin{aligned}
K_{\nu}^{\xi \xi^{\prime \prime}}(\mathbf{q}, \omega) & =-\nu \frac{H\left\langle S^{\zeta}\right\rangle}{E_{q+}^{2}-E_{q-}^{2}}\left[E_{\mathbf{q} \nu}^{2}-\lambda_{q}^{2}\right] f_{\nu}(\mathbf{q}, \omega), \\
K_{\nu}^{j j^{\prime \prime}}(\mathbf{q}, \omega) & =-\nu \frac{2 \lambda_{q}}{E_{q+}^{2}-E_{q-}^{2}}\left[E_{\mathbf{q} j \nu}^{2}-\varepsilon_{q}^{2}\right] f_{j \nu}(\mathbf{q}, \omega), \\
K_{\nu}^{j \xi^{\prime \prime}}(\mathbf{q}, \omega) & =-\nu \frac{2 \Phi_{\mathbf{q} j}^{*} \lambda_{q} H\left\langle S^{\zeta}\right\rangle \sin \varphi}{E_{q+}^{2}-E_{q-}^{2}} f_{j \nu}(\mathbf{q}, \omega) .
\end{aligned}
$$

The widths of the pseudospin-like $\left[\Gamma_{-}(\mathbf{q}, \omega) \equiv \Gamma_{q-}\right]$ and phonon-like $\left[\Gamma_{j+}(\mathbf{q}, \omega) \equiv\right.$ $\Gamma_{q+}$ ] modes [as being studied, for instance, in [14] (cf. also [5,7])] are introduced with the aid of the response function for the damped harmonic oscillator [27],

$$
f_{\nu}(\mathbf{q}, \omega)=\frac{\omega \Gamma_{\nu}(\mathbf{q}, \omega)}{\pi\left(\omega^{2}-E_{\mathbf{q} \nu}^{2}\right)^{2}+\left[\omega \Gamma_{\nu}(\mathbf{q}, \omega)\right]^{2}} .
$$

If, for the sake of simplicity, the local-potential asymmetry is neglected, the peculiar pseudospin, phonon and mixed form-factors in equations (28) are given by $[27,30]$ (cf. [5]):

$$
\begin{aligned}
F(\kappa)= & \beta_{\kappa} \cos \varphi-\mathrm{i} \alpha_{\kappa} \sin (\kappa \cdot \mathbf{l}) \sin \varphi \\
F(\kappa, \mathbf{q})= & \left\{\alpha_{\kappa} \cos [(\kappa-\mathbf{q}) \cdot \mathbf{l}]+2 \beta_{\kappa}\left\langle S^{\zeta}\right\rangle \sin \varphi \cos (\mathbf{q} \cdot \mathbf{l})\right. \\
& \left.+2 \mathrm{i} \alpha_{\kappa}\left\langle S^{\zeta}\right\rangle \cos \varphi \sin [(\kappa-\mathbf{q}) \cdot \mathbf{l}]\right\} \\
L_{j}(\kappa, \mathbf{q})= & (1 / 2) \alpha_{\kappa} \cos [(\kappa-\mathbf{q}) \cdot \mathbf{l}] F_{o j}(\kappa, \mathbf{q}), \\
\widetilde{F}_{j}(\kappa, \mathbf{q})= & F(\kappa, \mathbf{q}) F_{o j}(\kappa, \mathbf{q})\left|\Lambda_{\mathbf{q} \mathbf{j}}\right| \\
F_{o j}(\kappa, \mathbf{q})= & \mathrm{i}\left(\kappa \cdot \mathbf{e}_{\mathbf{q} \mathbf{j}}\right) / \sqrt{2 m_{j} \omega_{q j}} .
\end{aligned}
$$

The parameters $\alpha_{\kappa}$ and $\beta_{\kappa}$ depend on the model double-well potential. In the simplest model of a double-harmonic oscillator,

$$
\alpha_{\kappa}=\exp \left(-\kappa^{2} a_{\mathrm{c}}^{2} / 4\right), \quad \beta_{\kappa}=\alpha_{\kappa} \exp \left[-\left(l_{\mathrm{c}} / a_{\mathrm{c}}\right)^{2}\right]
$$


where $a_{\mathrm{c}}=\left(\hbar / m_{\mathrm{c}} \omega_{\mathrm{c}}\right)^{1 / 2}[29,30]$. If the asymmetry of the one-particle potential is determined only by the difference between the well depths, then it is automatically taken into account by the pseudospin formalism in the calculation of $\left\langle S^{x}\right\rangle=\left\langle S^{\xi}\right\rangle \sin \varphi$, $\left\langle S^{z}\right\rangle=\left\langle S^{\xi}\right\rangle \cos \varphi$, occurring in equations (25) and (31).

\subsection{Characteristics of elastic scattering}

The coherent elastic scattering exhibits sharp Bragg intensity peaks determined by the condition: $\hbar \kappa=\mathbf{p}^{\prime}-\mathbf{p}=\hbar \mathbf{b}$, and they are modulated by the pseudospin form factor (25) which strongly depends on the direction of the vector $\kappa$. The behavior peculiar to light ions (hydrogens) in double-well potentials (characterized by the interference effect) manifests itself most explicitly in the case of incoherent scattering. For sufficiently small $\kappa(\kappa \leqslant \pi / l)$ the phase factors $\exp \left(\mathbf{i} \mu \mathbf{q} \mathbf{l}_{\mu}\right)$ lead to a special periodic dependence in equations (19) and (20), i.e. to a characteristic "autocorrelating interference effect" due to neutron scattering on tunnelling light ions inside the single particle potentials. This has stimulated extremely thorough and subtle neutronographic investigations which enabled experimental verification of the contemporary physical concepts of the order-disorder ferroelectrics in general (cf. [5]). The temperature dependence of the scattering intensity is determined by the statistical averages $\left\langle S^{x}\right\rangle$ and $\left\langle S^{z}\right\rangle$. As it follows from equations (19) and (20), in the antiferroelectric phase $\left(T<T_{\mathrm{N}}\right)$ the expressive angular dependence vanishes, but the effective cross section increases. On the other hand, the temperature dependence (if one neglects small term proportional to $\left\langle S^{x}\right\rangle$ ) vanishes for the scattering vector $\kappa$ perpendicular to the direction of motion of light ions in the double-well potential. This allows for direct observation of the temperature dependence of the Debye-Waller factor in elastic incoherent scattering experiments.

The intensity of the corresponding coherent scattering is significantly enhanced on deuterated specimens. That makes possible the well-refined determination of the position and nature of the motion of the light atoms $(H$ or $D)$ in the family of hydrogen-containing compounds. Note also the significant contribution of the pseudospin-phonon interference term in equation (19), exhibiting quite complicated angular dependence which can be partially removed by an appropriate choice of the scattering geometry (for example $\kappa \perp \mathbf{l}_{\mu}$. At the same time, coherent elastic scattering on heavy ions is described by the usual structure factor, while incoherent elastic scattering (neglecting the Debye-Waller factor) is independent of the scattering angle and is similar to a background, as in the case of X-ray scattering.

In the antiferroelectric phase $\left(T<T_{\mathrm{N}}\right)$, as pointed in the previous section, one must take into account the pseudospin modulation (equation (15)) which leads to modification of the form-factors (25) [and (31), for the case of inelastic scattering] and accordingly the additional term proportional to $\Delta\left(\kappa+\mathbf{q}_{\mathrm{B}}\right)$ appears in equation (19). Almost the same relationship holds in the case of an incommensurate transition which, instead of wave vector on the boundary of $\mathrm{BZ}, \mathbf{q}_{\mathrm{B}}$, is described by the vector $\mathbf{q}_{M}\left(0<\mathbf{q}_{M}<\mathbf{q}_{\mathrm{B}}\right)$, corresponding to a given modulated structure. For incommensurate transitions the respective modulation of pseudospins $\left\langle S_{n r}^{z}\right\rangle=\left\langle S_{r}^{z}\right\rangle \exp \left(\mathbf{i q}_{M} \cdot \mathbf{n}\right)$ leads to the adequate changes owing to the redefinition of 
the effective form factor $F_{\mathrm{c}}(\kappa)(25)$. The main difference consists in the fact that, besides the Bragg reflections, there also arise peaks at $\kappa=\mathbf{b}-\mathbf{q}_{M}$, i.e. in the cross section corresponding to light ions only (index c) there appears the term

$$
\frac{1}{N}\left(\frac{\mathrm{d} \sigma_{\mathrm{ch}}}{\mathrm{d} \Omega}\right)_{\mathbf{q}_{M}}^{\mathrm{c}}=N \Delta\left(\kappa+\mathbf{q}_{M}\right)\left(b_{\mathrm{ch}}^{\mathrm{c}}\right)^{2}\left|\mathrm{e}^{-W_{\kappa}^{\mathrm{c}}} F_{\mathrm{c}}^{\prime \prime}(\kappa)\right|^{2},
$$

where $F_{\mathrm{c}}^{\prime \prime}(\kappa) \equiv \operatorname{Im} F_{\mathrm{c}}(\kappa)$. At incommensurate transitions, a more complicated modulation of the structure may take place. One of such situations is encountered in the crystal $\mathrm{NaNO}_{2}$ in which the nature of structural transitions is still unclarified (cf. $[1,4])$.

\subsection{Characteristics of the inelastic scattering}

Since the inelastic part of the scattering function for a heavy-ion subsystem is of the form usual for a phonon system, we shall consider only the parts which correspond to the dominant contribution of scattering on a light-ion system and to the interference term describing scattering by both subsystems. We note that no correlation functions are present in the scattering function $F_{q}^{\nu}(\kappa, \omega)(28)$ other than the phonon ones. This is the consequence of neglecting the effect of the motion of light ions on the vibrations of heavy ions which is physically justifiable. Hence, however, one cannot draw the conclusion that the motion of heavy ions is not anharmonic; just because of the coupling (sometimes very strong) between the two subsystems at a structural phase transition, the equilibrium positions of the heavy ions are noticeably shifted, leading to the macroscopic effect of spontaneous antipolarization arising in the system.

Unlike the "inertial" heavy-ion subsystem, as one can see from the expression $P_{q}^{\nu}(\kappa, \omega)(28)$, scattering on the "active" light-ion subsystem is determined, besides pseudospin correlators, by correlators of the phonon and mixed types as well. In the local-normal-coordinate and in the related cell-pseudospin-variable representation such contributions of correlators were obtained in [7] and, subsequently, with account of the real structure in [27] (for describing scattering processes of neutrons in ferroelectrics and antiferroelectrics with hydrogen bonds). It is easy to demonstrate that in the local-normal-coordinate representation the part of the scattering function $P_{q}^{\nu}(\kappa, \omega)$ connected with the phonon correlator, if the asymmetry in the form factors (31) is neglected, has a form similar to that of the scattering function on phonons with the effective cross sections:

$$
\left(b_{\mathrm{ch}}^{c j}\right)^{2}=\left(b_{\mathrm{ch}}\right)^{2}\left|\tilde{F}_{j}(\kappa, \mathbf{q})\right|^{2} ; \quad\left(b_{\mathrm{ic}}^{c j}\right)^{2}=\left(b_{\mathrm{ic}}\right)^{2}\left|\tilde{F}_{j}(\kappa, \mathbf{q})\right|^{2},
$$

provided that for the symmetric wells vibrating in unison with the center of their joining distance holds $\Lambda_{q j}(+)=\Lambda_{q j}(-) \equiv \Lambda_{q j}$. Consequently, the scattering processes described by the function $K_{j j^{\prime}}^{\prime \prime}(\mathbf{q}, \omega)$ in $P_{q}^{\nu}(\kappa, \omega)$ are related to the energy transfer, lost by the neutrons, from the light-ion subsystem to the heavy ions (lattice). This energy is transferred from the pseudospin mode to the phonon modes of the system ["(anti)ferroelectro-vibrational scattering"] [25]. Such scattering may 
sometimes turn out to be more convenient for determining the phonon frequency distribution function, at least in the paraelectric phase (cf. [5,27]).

In the antiferroelectric phase, as pointed out in the section 2 , all summations over $q$ have to be substituted by $\left[\sum_{q} f(q)+f\left(q+q_{\mathrm{B}}\right)\right]$ over new BZ. The modifications in the form factor $\tilde{F}(\kappa, \mathbf{q})$ (i.e. $F(\kappa, \mathbf{q}))$ take place and accordingly in the scattering terms $P_{q}^{\nu}(\kappa, \omega)$ and $I_{q}^{\nu}(\kappa, \omega)$. Thus, the additional peaks around $\kappa=\mathbf{b}-\mathbf{q}_{\mathrm{B}}$ (modulation terms) appear, which, in common with autocorrelation and interaction interference terms, brings us to very complex but more proper expressions to be used in fitting the experimental data.

\section{Comments and possible applications}

Theoretical and experimental problems in the examination of antiferroelectrics and ferroelectrics of the order-disorder type are long standing ones and there is no substantial progress in their resolving during the last few years. Here we recall some of them assuming that they may be overcome within the framework of the improved and the adjusted dynamical model of pseudospin-phonon interaction and its application in more sophisticated neutronografic analysis. Herein below we list a few most characteristic examples.

(I) Application to ADP $\left(\mathrm{NH}_{4} \mathbf{H}_{2} \mathbf{P O}_{4}\right)$ : Besides the results similar to the ones for hydrogen-bonded ferroelectrics $\left(\mathrm{KH}_{2} \mathrm{PO}_{4}\right.$ and its family), the difficulties related to the presence of a significant contribution to the incoherent neutron scattering from $\mathrm{NH}_{4}$ molecules and to the assignment of acoustical, optical, torsional and librational motions of all ions in the system are noted (cf. [5]). Also, the existence of the $\mathrm{ND}_{4}$-librational soft mode coupled with deuteron fluctuations on O... D-O bonds (by both incoherent neutron scattering and deuteron NMR [34]) as well as of an over-damped antiferroelectric-soft mode (by quasi-elastic coherent neutron scattering, in the vicinity of the point (504) at the reciprocal lattice space and $T=244 \mathrm{~K}$ [35]) was observed. However, a detailed (near "satellite" reflections) analysis of ion displacements (and of their symmetry) should be carried out as relied on both the improved theoretical predictions (just as the presented ones) and on the experimental refinements (cf. [5]).

(II) Application to Rochelle salt $\left(\mathrm{NaKC}_{4} \mathrm{H}_{4} \mathrm{O}_{6} \cdot \mathbf{4} \mathrm{H}_{2} \mathrm{O}\right)$ : The situation in this antiferroelectric is much more complicated (cf. [2,5,36]). Although several peaks as attributed to $\mathrm{H}$-vibrations were indicated (in inelastic neutron scattering), only two of them converged significantly on the transition from non-polar to polar (ferrielectric) phase being attributed to the shortest hydrogen bonds. In accord to structural data $[2,36]$ the tunnelling motion could be attributed to protons within $\mathrm{O}_{(1)}-\left(\mathrm{H}_{2} \mathrm{O}\right)_{(10)}$ bonds or $(\mathrm{OH})_{(5)}$ dipoles and in some extent to water molecules $\left(\mathrm{H}_{2} \mathrm{O}\right)_{(8)}$. Tentatively such dipoles could be coupled with heavy ions of distorted octahedra of tartrate groups $\left(\mathrm{C}_{2} \mathrm{O}_{6}\right)$ in common with Na- and K-ions. Based on more reliable structural data and on 
refined experimental studies, neutron scattering intensities should be fitted by the expressions presented, using very successful numerical estimations as given in $[15,18]$. In such a way, the controversy about the nature (i.e. the origin) of dipoles and in what kind of a double-well potential they tunnel might be overcome.

(III) Application to $\mathrm{NaNO}_{2}$ : Although the structure of $\mathrm{NaNO}_{2}$ is simple in comparison to other order-disorder ferroelectrics, this crystal is probably the most complex non-hydrogenous substance for which a rather complete neutronographic investigation of vibrational modes has been undertaken [1]. The measurements of critical dynamics show that changes in ferroelectric fluctuations are much slower than any vibrations of a phonon subsystem and involve large displacements of all the atoms in the cell. This resembles the feature of the double-well local-mode model as discussed heretofore. For the analysis of such long-wavelength fluctuations the other experimental techniques have been employed (cf. [1]). However, there is no direct confirmation of the mode softening, neither of the phonon nor of the tunnelling type. There were also many contradictions in assigning the rotational flipping around the $a$ - or $c$-axis. Furthermore, it is still unclear whether and when one of these two possibilities prevails. Unlike the neutronographic studies, in X-ray scattering the appearance of the antiferroelectric configuration of the sinusoidal pattern near $T_{\mathrm{N}} \approx 437.5 \mathrm{~K}$ has been observed closer to $\mathbf{q}=\mathbf{q}_{M}=\mathbf{a}^{*} / 8$, moving towards the theoretically predicted value $\mathbf{a}^{*} / 5$ (cf. [1]). Thus, although there was no clear evidence in favor of another subtle (rather exotic) polar phase, which may exist between ferroelectric and the sinusoidal antiferroelectric phase, some deeper insight has been gained in the qualitative understanding of the respective competition of the latter two phases.

In order to overcome numerous confusions concerning the nature of phase transitions in $\mathrm{NaNO}_{2}$, a part of which was stated above, the critical dynamics in this crystal could be described by the pseudospin variables associated either with the large-scale motion of nitrogen atoms of each $\mathrm{NO}_{2}$-group or with the slow librational reorientations of the $\mathrm{NO}_{2}$-groups themselves (along the $b$-axis) (cf. $[1,4]$. The picture is completed by the coupling (probably not too rigid) between the flipping of nitrite ions (or groups as a whole) and the displacements of sodium ions (possibly of nitrite groups as well). Thus, a very complex pseudospin-phonon model should be postulated and applied in the dynamic neutronographic examinations in accordance with the theory presented.

(IV) Application to high- $T_{\mathrm{c}}$ superconducting copper-oxide compounds: The pseudospin model of Rochelle salt-type (associated with the apical oxygen O4) was developed several years ago (cf. [19-21] and references therein). It was supplemented by Müller's (electron-pseudospin) "field" $\left[H_{i}^{z}=g \sum_{l}\left\langle n_{i+l}\right\rangle\right.$ - representing the role of electron carrier density $n_{i+l}$ of four oxygens (index l) in $\mathrm{CuO}_{2}$ sheet near the $i$-th $\left.\mathrm{O} 4\right]$ as well as by the coupling of the O4tunnelling motion with $\mathrm{Cu}_{2}-\mathrm{O}_{2}, \mathrm{O}_{3}$-vibrations (modes) of $\mathrm{A}_{g^{-}}$or $\mathrm{B}_{g^{-}}$-symmetry 
(analogous to $E_{q j+}$ modes) $[19,21,33]$. Unlike the present neutron scattering analysis, it is tempting to conclude that the well-enough refined cross sections [equations (18-31)] could be applied to dismiss many controversies in testing the existence of structural, dielectric and charge instabilities, asymmetry of the local $\mathrm{Cu}_{2}-\mathrm{O} 4-\mathrm{Cu}_{2}$ potential, vibronic states, etc., up to the problem of the mutual origin of superconducting and (anti)ferroelectric phases, i.e. their possible coexistence. As for the adjustment of the cross sections presented, it might be in the fashion of papers devoted to the studies of some interference and unusual effects in $\mathrm{LaBaCuO}$ and $\mathrm{YBaCuO}$ compounds [37] as observed in neutron scattering experiments (cf. [19-21]).

Concluding this paper it should be emphasized that although it is mainly confined to the neutron scattering properties of hydrogen-bonded antiferroelectrics, it is clear enough that the derived expressions hereto, as based on the pseudospin-phonon formalism and its extensions, do hold equally well for diverse physical systems when suitable adaptations and interpretations are made.

\section{Acknowledgement}

I would like to express my gratitude to Academician Igor V. Stasyuk for his invitation to contribute to the special issue of Condensed Matter Physics in tribute of 60th anniversary of Professor Roman R. Levitskii. I also acknowledge Mr. V.Stojanović, Dr. Z.Ivić and Dr. J.Raičević for their help in preparing this manuscript.

This work was supported by the Serbian Ministry of Science and Technology under the contract number 1961.

\section{References}

1. Lines M.E., Glass A.M. Principles and Applications of Ferroelectrics and Related Materials. Oxford, Clarendon Press, 1977.

2. Blinc R., Žekš B. Soft Modes in Ferroelectrics and Antiferroelectrics. Amsterdam, North Holland, 1974.

3. Vaks V.G. Introduction to the Microscopic Theory of Ferroelectrics. Moscow, Nauka, 1973 (in Russian).

4. Bruce A.D., Cowley R.A. Stuctural Phase Transition. London, Taylor and Francis Ltd., 1981.

5. Aksenov V.L., Plakida N.M., Stamenković S. Neutron Scattering by Ferroelectrics. Singapore, World Scientific, 1989.

6. Thomas H. Structural Phase Transitions and Soft Modes. - In: Structural Phase Transitions and Soft Modes. Eds. Samuelsen E.J., Andersen E. and Feder J. Oslo-BergenTromso, Universitetsforlaget, 1971, p. 15-45.

7. Moore M.A., Williams H.C.W.L. // J. Phys. C, 1972, vol. 5, No. 22, p. 3168-3184.

8. De Gennes P.G. // Solid State Commun., 1963, vol. 1, p. 132-137.

9. Villain J., Stamenković S. // Phys. Stat. Solidi, 1966, vol. 15, p. 585-596.

10. Kobayashi K.K. // J. Phys. Soc. Japan, 1968, vol. 24, No. 3, p. 497-508. 
11. Stasyuk I.V., Levitskii R.R. // Ukr. Fiz. Zhurn., 1970, vol. 15, No. 3, p. 460-469 (in Russian).

12. Stasyuk I.V., Levitskii R.R. // Phys. Stat. Solidi, 1970, vol. 39, p. K35-K37; Stasyuk I.V. // Teor. Mat. Fiz., 1971. vol. 9, No. 3, p. 431-439.

13. Stasyuk I.V., Kaminskaya N.M. // Ukr. Fiz. Zhurn., 1974, vol. 19, No. 2, p. 237-243 (in Russian); // Ukr. Fiz. Zhurn., 1974, vol. 19, No. 2, p. 243-252 (in Russian).

14. Levitskii R.R., Kozitskii Yu.V. Preprint of the Institute of Theoretical Physics, ITP74-108P, Kiev, 1974 (in Russian); Levitskii R.R., Sorokov S.T. Preprint of the Institute of Theoretical Physics, ITP-77-53P, Kiev, 1977, 43 p. (in Russian).

15. Konsin P.I. // Phys. Stat. Solidi (b), 1975, vol. 70, p. 451-459.

16. Aksenov V.L., Schreiber J. // Phys. Stat. Solidi (b), 1977, vol. 81, p. 371-378.

17. Blinc R., Žekš B. // J. Phys. C, 1982 vol. 15, No. 22, p. 4661-4670.

18. Žekš B., Shukla G.C., Blinc R. // Phys. Rev. B, 1971, vol. 3, No. 7, p. 2306-2309.

19. Plakida N.M. High-Temperature Superconductors. Berlin, Springer, 1995.

20. Galbaatar T., Plakida N.M., Drechsler S.L. Preprint JINR E17-94-299, Dubna, 1994, $16 \mathrm{p}$.

21. Stasyuk I.V., Shvaika A.M. // Acta Physica Polonica A, 1993, vol. 84, No. 2, p. 293313; Ferroelectrics, 1997, vol. 192, p. 1-10.

22. Montgomery H., Paul G.L. // Proc. Roy. Soc. (Edinbrough), 1972, vol. 70, No. 11, p. $107-124$.

23. Stasyuk I. V., Levitskii R.R. // Fiz. Elektronika, 1970, vol. 2, p. 3-11 (in Ukrainian).

24. Stamenković S. A quasi-spin model and frequency spectrum in $\mathrm{NH}_{4} \mathrm{PO}_{4}$ antiferroelectric crystal. - In: Book of Abstracts of Europ. Meeting on Ferroelectricity, Saarbrücken, March 27-29, 1989, p. 6.

25. Stasyuk I.V., Levitskii R.R. // Izv. Akademii Nauk SSSR, 1971, vol. 35, No. 9, p. 17751778 (in Russian).

26. Stamenković S. On the neutron scattering in $\mathrm{KH}_{2} \mathrm{PO}_{4}$ ferroelectric crystal. - In: Book of Abstracts of Europ. Meeting on Ferroelectricity, Saarbrücken, March 27-29, 1969, p. 6 .

27. Levitskii R.R., Perekresnii S.M. Preprint of the Institute of Theoretical Physics, ITP79-59P, 1979, Kiev, 43 p. (in Russian); Preprint of the Institute of Theoretical Physics, ITP-80-15P, 1980, Kiev, 31 p. (in Russian); Preprint of the Institute of Theoretical Physics, ITP-80-16P, 1980, Kiev, 31 p. (in Russian).

28. Stamenković S. Single-particle potential assymetry effects in the theory of the neutron scattering by order-disorder feroelectrics (to be published).

29. Aksenov V.L., Stamenković S. // Solid State Physics, 1979, vol. 19, No. 8, p. 13661372 (in Russian).

30. Stamenković S. // Journal Low Temp. Phys., 1972, vol. 9, No. 5/6, p. 475-483; ibid. p. 485-493.

31. Ribeiro L.A.A., Pires A.S.T., Barreto F.C. // Ferroelectrics, 1981, vol. 31, p. 1-7.

32. Cochrane W. // Adv. Phys., 1969, vol. 18, p. 157-192.

33. Stamenković S. Interferrence effects and polar modulation in dynamical neutronography of hydrogen-bonded antiferroelectrics. - In: Proccedings of the National Symposium of Condensed Matter Physics, Arandjelovac, October 5-11, 2001 (in press).

34. Dimic V., Osredkar M., Slak J., Kandusar A. // Phys. Stat. Solidi (b), 1973, vol. 59, p. $471-478$.

35. Meister H., Skalyo J., Jr., Frazer B.C., Shirane G. // Phys. Rev., 1969, vol. 184, 


\section{p. $550-555$.}

36. Zheludev I.S. Foundations of ferroelectricity. Moscow, Atomizdat, 1973 (in Russian); Jona F., Shirane G. Ferroelectric Crystals. Moscow, Mir, 1965 (in Russian).

37. Plakida N.M., Aksenov V.L., Drechsler S.L., Galbaatar T., Stamenković S. // Modern Physics Lett. B, 1990, vol. 4, No. 5, p. 341-346; Plakida N.M., Stamenković S. // Modern Physics Lett. B, 1991, vol. 5, No. 10, p. 717-719.

\section{Особливості розсіювання нейтронів антисегнетоелектриками типу лад-безлад}

\section{С.Стаменкович}

Інститут ядерних досліджень "Вінча", Югославія, Сербія, Белград

Отримано 7 серпня 2002 р., в остаточному вигляді - 25 серпня $2002 \mathrm{p}$.

З використанням динамічної протон (псевдоспін)-іонної (ґратка) моделі та формалізму функцій Гріна, розраховані ефективні повні диференціальні перерізи для когерентного і некогерентного розсіювання нейтронів. Характерні інтерференційні ефекти, пов'язані як з тунелюванням легких іонів (протонів), так і зі змішаними розсіювальними процесами протон-важкий іон, виражені через відповідні псевдоспінові і псевдоспін-фононні форм-Фактори. Крім того, загальний вигляд інтенсивності розсіювання в антисегнетоелектричній фазі визначається також ефектами модуляції поляризації та додатковою інтерференцією у перерізах розсіяння. Передбачення теорії обговорюється у зв'язку з експериментальними дослідженнями кристалів ADP, сегнетової солі і $\mathrm{NaNO}_{2}$. Коротко обговорюється їх можливе застосування для перевірки співіснування (анти)сегнетоелектричної та надпровідної фаз у високотемпературних надпровідниках на основі оксиду міді.

Ключові слова: розсіювання нейтронів, антисегнетоелектрики, псевдоспін-фононна модель

PACS: $61.12 . B t$ 